\title{
New records of long-legged flies (Diptera: Dolichopodidae) for Iran
}

\section{Новые указания мух-зеленушек (Diptera: Dolichopodidae) из Ирана}

A. Ahmadi ${ }^{1}$, M. Gheibi ${ }^{1}$, H. Ostovan ${ }^{1}$, S. Hesami ${ }^{1}$, I.Ya. Grichanov ${ }^{2}$

А. Ахмади ${ }^{1}$, М. Гейби ${ }^{1}$, Х. Остован ${ }^{1}$, С. Хезами ${ }^{1}$, И.Я. Гричанов ${ }^{2}$

\footnotetext{
${ }^{1}$ Department of Entomology, Faculty of Agricultural Sciences, Shiraz Branch, Islamic Azad University, Shiraz, Iran. E-mail: gheybi@iaushiraz.ac.ir

${ }^{2}$ All-Russian Institute of Plant Protection, Podbelskogo 3, 196608, St.Petersburg-Pushkin, Russia. E-mail: grichanov@mail.ru

${ }^{2}$ Всероссийский институт защиты растений, шоссе Подбельского 3, Санкт-Петербург-Пушкин 196608, Россия.
}

KEY WORDS: Dolichopodidae, Iran, Markazi province, Lorestan province, new records.

КЛЮЧЕВЫЕ СЛОВА: Dolichopodidae, Иран, Меркези, Лурестан, новые указания.

ABSTRACT. During a 2016 survey conducted in Markazi and Lorestan provinces located in the Central Iran, 1490 specimens of Dolichopodidae were collected and identified. Two genera (Lamprochromus sp. and Micromorphus sp.) and eight dolichopodid species [Chrysotus pennatus Lichtwardt, 1902, Sybistroma impar (Rondani, 1843), Tachytrechus beckeri Lichtwardt, 1917, Medetera pallipes (Zetterstedt, 1843), Campsicnemus armatus (Zetterstedt, 1849), C. magius (Loew, 1845), C. tomkovichi Grichanov, 2009, and Syntormon fuscipes (von Roser, 1840)] are recorded for the first time in Iran.

РЕЗЮМЕ. В 2016 году в останах Меркази и Лурестан, расположенных в Центральном Иране, были собраны и определены 1490 экземпляров Dolichopodidae. Два рода (Lamprochromus sp. и Micromorphus sp.) и восемь видов мух-зеленушек [Chrysotus pennatus Lichtwardt, 1902, Sybistroma impar (Rondani, 1843), Tachytrechus beckeri Lichtwardt, 1917, Medetera pallipes (Zetterstedt, 1843), Campsicnemus armatus (Zetterstedt, 1849), C. magius (Loew, 1845), C. tomkovichi Grichanov, 2009, и Syntormon fuscipes (von Roser, 1840)] оказались новыми для Ирана.

\section{Introduction}

Approximately 7600 described species and 250 genera of the long-legged flies (Dolichopodidae) are known in the world fauna [Grichanov, 2014]. The most recent Iranian list of these flies includes 114 species collected mainly from the north-west of Iran [Grichanov, 2016]. Available information about Dolichopodidae of the Central Iranian Markazi and Lorestan provinces has not been known before our investigations. Grichanov et al. [2010] and Ahmadi et al. [2016] published original data on 14 species collected in the Markazi province and seven species from the Lorestan province.
Material and methods

During 2016 season 1490 specimens have been sampled from 64 sites in the Markazi and Lorestan provinces mostly by standard sweep net. Some specimens have been collected by use of yellow pan traps and an exhauster mentioned at material listed below. All the identified species are included in the keys to more than 500 Dolichopodidae species of the Caucasus and Eastern Mediterranean [Grichanov, 2007]. The last author has checked in St. Petersburg (Russia) all the identifications made by the first author. The Caucasian and general species distribution is given after Negrobov et al. [2013] and Grichanov [2014]. Type localities are provided, and country lists (in Asia) are arranged alphabetically. Sampling data include: location, coordinates, altitude ( $\mathrm{m}$ a.s.1.), and dominant plant community. The collector of all specimens is the first author of this paper; her name is omitted from the species list. Photos of some species newly recorded from Iran are provided. The specimens are deposited in ethyl alcohol in Shiraz Branch, Islamic Azad University, Shiraz, Iran.

\section{Species list}

Subfamily Diaphorinae Schiner, 1864 Argyra Macquart, 1834

Argyra leucocephala (Meigen, 1824)

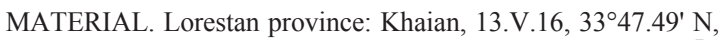
48 54.44' E, $1676 \mathrm{~m}$, on Hordeum murinum near wheat fields, $10^{7}$ '.

DISTRIBUTION. Type locality: not given [Europe]. Caucasus: Azerbaijan, Russia (Adygea, Chechnya, Krasnodar Territory). Europe, North Africa, Asia (Iran, Israel, Turkey).

Chrysotus Meigen, 1824

Chrysotus obscuripes Zetterstedt, 1838

MATERIAL. Markazi province: Seyed Esmaeel road, 22.V.16, $34^{\circ} 14.24^{\prime}$ N, $49^{\circ} 08.31^{\prime}$ E, 2027 m, on Poaceae and Juncus sp., $10^{\prime}$ '. 
DISTRIBUTION. Type locality: Sweden: Lapponica Umensi, Fredrica. Caucasus: Armenia. Europe, Asia (Palaearctic China, Iran, Kyrgyzstan, Russian Far East, Siberia, Turkey).

REMARK. Khaghaninia et al. [2016] mentioned this species for Iran, not giving original material.

\section{Chrysotus pennatus Lichtwardt, 1902}

Fig. 4.

MATERIAL. Lorestan province: Dareh Mahee, 11.VII.16, $33^{\circ}$ 01.44' N, 49 38.41' E, $1810 \mathrm{~m}$, on Juncus sp. and Typha sp., $10^{7}$.

DISTRIBUTION. Type locality: Bosnia and Herzegovina: Novi. Caucasus: Armenia, Russia (Adygea, Krasnodar Territory). Europe, Asia (Palaearctic China, Turkey). New record for Iran.

\section{Chrysotus suavis Loew, 1857}

MATERIAL. Lorestan province: Aligoudarz, 11.VII.16, $33^{\circ}$ $22.59^{\prime} \mathrm{N}, 49^{\circ} 43.39^{\prime} \mathrm{E}, 2024 \mathrm{~m}$, on Typha sp., 50 $30^{7}, 3$.

DISTRIBUTION. Type locality: Germany: "Coln"; Austria: "Neusiedler See in Ungarn". Caucasus: Armenia, Azerbaijan, Georgia, Russia (Adygea, Alania, Kabardino-Balkaria, Krasnodar Territory, Rostov Region). Europe, North Africa, Asia (Afghanistan, Palaearctic China, Iran, Iraq, Israel, Kyrgyzstan, Middle Asia, Mongolia, Russian Far East, Siberia, Turkey)

Subfamily Dolichopodinae Latreille, 1809 Dolichopus Latreille, 1796

Dolichopus griseipennis Stannius, 1831

MATERIAL. Lorestan province: Khaian, 13.V.16, 3347.49' N, $48^{\circ} 54.44^{\prime} \mathrm{E}, 1676 \mathrm{~m}$, on Hordeum murinum near wheat fields, 1 \%

DISTRIBUTION. Type locality: France: "Lyon”. Caucasus: Armenia, Azerbaijan, Georgia, Russia (Adygea, Chechnya, Krasnodar Territory). Europe, North Africa, Asia (Cyprus, Iran, Israel, Kazakhstan, Turkey, "Middle Asia", "Siberia").

Dolichopus perversus Loew, 1871

MATERIAL. Markazi province: Hossein Abad, 19.V.16, $34^{\circ}$ $01.43^{\prime}$ N, 49 $46.28^{\prime}$ E, 1915 m, on Juncus sp. and Equistum sp., $10^{7}$

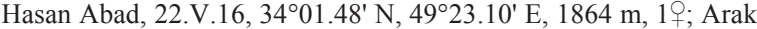
19.VI.16, 34 ${ }^{\circ} 04.67^{\prime} \mathrm{N}, 49^{\circ} 43.36^{\prime} \mathrm{E}, 1643 \mathrm{~m}, 20^{\top}, 1$, 1 (Yellow pan

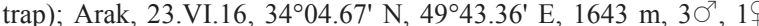
(Yellow pan trap); Lorestan province: Jooshan, 11.VII.16, $33^{\circ} 21.43$ N, 4944.40' E, $2040 \mathrm{~m}$, on Typha sp., $80^{7}, 11$.

DISTRIBUTION. Type locality: Tajikistan: Zarawschan Thal, Turkestan [=Zeravshan valley]. Caucasus: Abkhazia, Armenia. Asia (Iran, Israel, Kazakhstan, Kyrgyzstan, Tajikistan, Turkey).

\section{Dolichopus siculus Loew, 1859}

MATERIAL. Markazi province: Gezerdar, 22.V.16, 34 $14.24^{\prime}$ N, 4908.31' E, 2027 m, $10^{\prime}$; Eijan, 22.V.16, 34 ${ }^{\circ} 16.89^{\prime} \mathrm{N}, 49^{\circ} 17.46$ E, $1765 \mathrm{~m}, 10^{7}$; Lorestan province: Khak Patieh, 3.VI.16, $33^{\circ} 07.83^{\prime}$

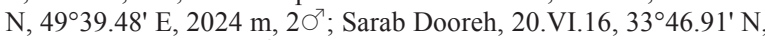

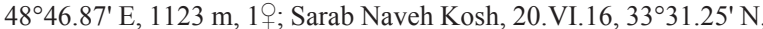

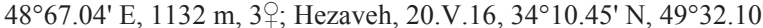
E, $1926 \mathrm{~m}$, on Poaceae and Equistum sp., $10^{7}$.

DISTRIBUTION. Type locality: Italy: Sidy. Europe (Bulgaria, France, Italy), Asia (Golan Heights, Iran, Israel).

REMARK. Grichanov's [2007] key has a misprinting in a couplet 84 (p. 108); in fact, the dorsal tooth is present on hypandrium of $D$. siculus and absent on hypandrium of D. excises (Pārvu, 1996). Therefore, records of the latter species in Iran [Grichanov et al., 2010] should be confirmed.
Dolichopus signifer Haliday, 1832

MATERIAL. Markazi province: Baghdadi, 19.V.16, 3401.75' N, 49 45.21' E, 2017 m, on Populus sp. (Trees), 20', 1; Hezaveh, 20.V. $16,34^{\circ} 10.45^{\prime} \mathrm{N}, 49^{\circ} 32.10^{\prime} \mathrm{E}, 1926 \mathrm{~m}$, on Poaceae and Equistum sp.,

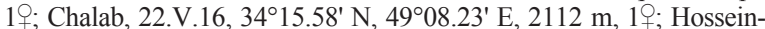

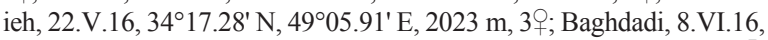
$34^{\circ} 02.05^{\prime} \mathrm{N}, 49^{\circ} 45.11^{\prime} \mathrm{E}, 2000 \mathrm{~m}$, on Juncus sp. and Equistum sp., $30^{\top}$, 1울 Hossein Abad, 8.VI.16, 3401.44' N, 4946.34' E, 1908 m, on Juncus sp. under Populus sp. (Trees), $10^{\top}$; Lorestan province: Jooshan, 11.VII.16, 33 $21.43^{\prime} \mathrm{N}, 49^{\circ} 44.40^{\prime} \mathrm{E}, 2040 \mathrm{~m}$, on Typha sp., 2 .

DISTRIBUTION. Type locality: Ireland: Roundstone Bay. Caucasus: Georgia, Russia (Chechnya, Kabardino-Balkaria, Krasnodar Territory, Rostov Region). Europe, North Africa, Asia (Afghanistan, Iran, Kazakhstan, Tajikistan, Turkey, Turkmenistan, Uzbekistan).

\section{Gymnopternus Loew, 1857}

\section{Gymnopternus flavitibia}

Pollet, Khaghaninia, Kazerani, 2017

MATERIAL. Markazi province: Gezerdar, 22.V.16, 34 $12.50^{\prime}$ $\mathrm{N}, 4^{\circ} 08.14^{\prime} \mathrm{E}, 2255 \mathrm{~m}, 1 \mathrm{O}^{7}, 1$.

DISTRIBUTION. Type locality: Iran: East Azerbaijan, Arasbaran, Keleybar. Asia (Iran).

\section{Hercostomus Loew, 1857 Hercostomus sp.}

MATERIAL. Markazi province: Noubaran, 28.V.14, $35^{\circ} 07.07^{\prime}$ N, $49^{\circ} 41.38$ E, 1645 m, on Brassica napus, 1 ㅇ ; Gheitanieh, 22.V.14, $35^{\circ} 02.24^{\prime} \mathrm{N}, 50^{\circ} 02.04^{\prime} \mathrm{E}, 1346 \mathrm{~m}$, on Hordeum sp., 1 ㅇ.

\section{Poecilobothrus Mik, 1878 Poecilobothrus sp.}

MATERIAL. Markazi province: Hak, 10.VII.15, 34 $59.58^{\prime} \mathrm{N}$, $55^{\circ} 22.06$ E, $1900 \mathrm{~m}$, on Juncus sp. and Salix sp. (Trees), 1 ㅇ․

\section{Poecilobothrus regalis (Meigen, 1824)}

MATERIAL. Markazi province: Gavgodar, 27.V.16, $34^{\circ} 06.45^{\prime}$ $\mathrm{N}, 49^{\circ} 21.80^{\prime} \mathrm{E}, 1826 \mathrm{~m}, 2$; ; Hak Olya, 15.VII.16, 33ํำ.91' N, $48^{\circ} 46.87^{\prime} \mathrm{E}, 1830 \mathrm{~m}, 60^{\top}, 10$ 웅 Lorestan province: Bisheh waterfall, 2.VI.16, $33^{\circ} 20.03^{\prime} \mathrm{N}, 48^{\circ} 52.79^{\prime} \mathrm{E}, 1212 \mathrm{~m}$, on Poaceae and Quercus

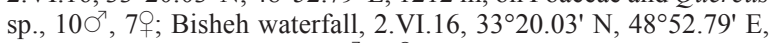
$1212 \mathrm{~m}$, on Ouercus sp., 20, 19 (Yellow pan trap); Ab Sefid

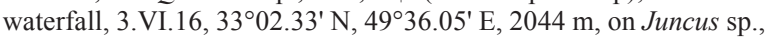

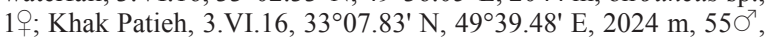

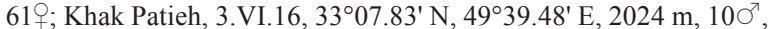
10; Shirvan, 20.VI.16, 32 ${ }^{\circ} 59.93^{\prime} \mathrm{N}, 49^{\circ} 35.28^{\prime} \mathrm{E}, 1456 \mathrm{~m}$, on Beta

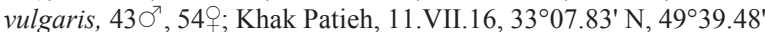
E, $2061 \mathrm{~m}$, on Poaceae and Typha sp., 507, 12; Khak Patieh,

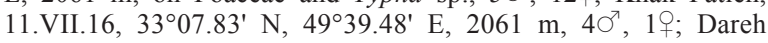

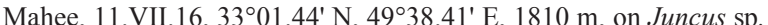

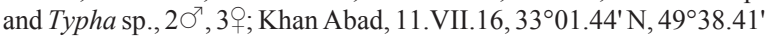

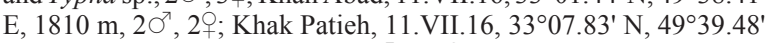

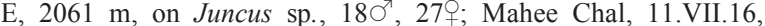
$33^{\circ} 08.33^{\prime} \mathrm{N}, 49^{\circ} 40.47^{\prime} \mathrm{E}, 1991 \mathrm{~m}$, on Juncus sp. and Typha sp. under Salix sp. (Trees), $3 \sigma^{\top}, 1$.

DISTRIBUTION. Type locality: not given. Caucasus: Azerbaijan, Georgia, Russia (Chechnya, Kabardino-Balkaria, Krasnodar Territory, Rostov Region, Stavropol Territory). Europe, Asia (Iran, Turkey, Uzbekistan).

Sybistroma Meigen, 1824 Sybistroma impar (Rondani, 1843) Figs 5-6.

MATERIAL. Markazi province: Arak, 8.VI.16, $34^{\circ} 04.67^{\prime} \mathrm{N}$, $49^{\circ} 43.36^{\prime}$ E, $1643 \mathrm{~m}$, on Juncus sp. and Avena sativa, $10^{\top}$.

DISTRIBUTION. Type locality: Italy. Caucasus: Russia (Chechnya, Krasnodar Territory). Europe, Asia (Israel, Turkey). New record for Iran. 

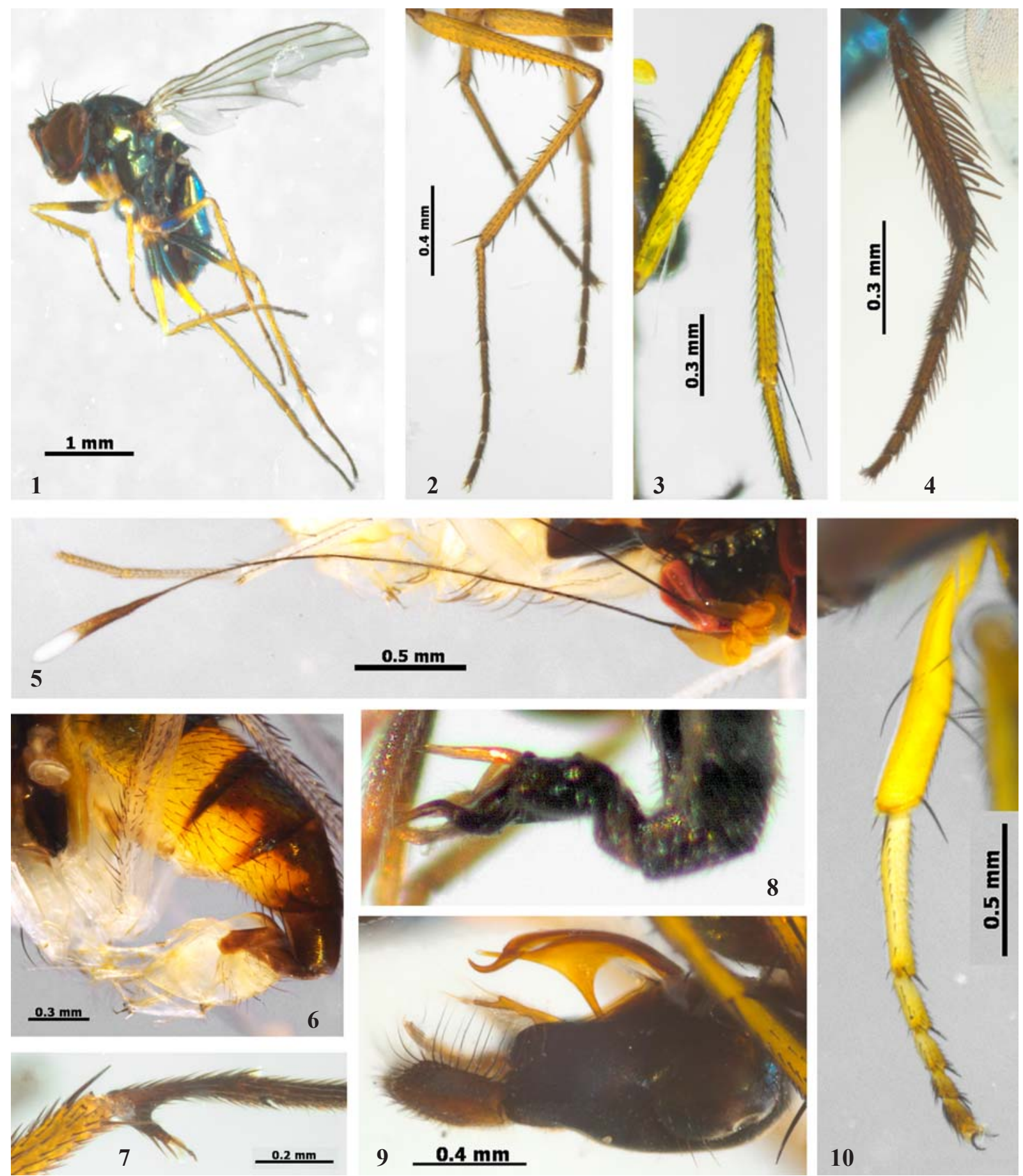

Figs 1-9. Diagnostic characters of Dolichopodidae species firstly found in Iran: 1 - Campsicnemus magius (Loew, 1845), female habitus; 2 - Campsicnemus armatus (Zetterstedt, 1849), male mid leg; 3 - Campsicnemus tomkovichi Grichanov, 2009, male hind femur and tibia; 4 - Chrysotus pennatus Lichtwardt, 1902, male hind tibia and tarsus; 5 - Sybistroma impar (Rondani, 1843), male antenna; 6 - S. impar, male abdomen; 7 - Syntormon fuscipes (von Roser, 1840), male hind basitarsus; 8 - Medetera pallipes (Zetterstedt, 1843), hypopygium; 9 - Tachytrechus beckeri Lichtwardt, 1917, hypopygium; $10-T$. beckeri, male fore tibia and tarsus.

Рис. 1-9. Диагностические признаки новых для Ирана видов Dolichopodidae: 1 - Campsicnemus magius (Loew, 1845), внешний вид самки; 2 - Campsicnemus armatus (Zetterstedt, 1849), средняя нога самца; 3 - Campsicnemus tomkovichi Grichanov, 2009, заднее бедро и голень самца; 4 - Chrysotus pennatus Lichtwardt, 1902, задняя голень и лапка самца; 5 - Sybistroma impar (Rondani, 1843), усик самца; 6 - S. impar, брюшко самца; 7 - Syntormon fuscipes (von Roser, 1840), 1-й членик задней лапки самца; 8 - Medetera pallipes (Zetterstedt, 1843), гипопигий; 9 - Tachytrechus beckeri Lichtwardt, 1917, гипопигий; 10 - T. beckeri, передняя голень и лапка самца. 
Sybistroma nodicornis Meigen, 1824

MATERIAL. Markazi province: Seyed Esmaeel road, 22.V.16, $34^{\circ} 13.68^{\prime} \mathrm{N}, 49^{\circ} 08.77^{\prime} \mathrm{E}, 2073 \mathrm{~m}, 6 \mathrm{O}^{\top}, 2$, ; Chalab, 22.V.16, 34 ${ }^{\circ} 15.58^{\prime}$ $\mathrm{N}, 4^{\circ} 08.23^{\prime} \mathrm{E}, 2112 \mathrm{~m}, 1^{\circ}$; Lorestan province: Ab Sefid waterfall, 3.VI.16, 3259.80' N, 49³5.17' E, 2127 m, 1 \% (exhauster).

DISTRIBUTION. Type locality: "not given". Europe, North Africa, "South Russia”, Asia (Iran, Iraq, Turkey).

Tachytrechus Haliday, 1851

Tachytrechus beckeri Lichtwardt, 1917 Figs 9-10.

MATERIAL. Lorestan province: Bisheh waterfall, 2.VI.16, $33^{\circ} 20.03^{\prime} \mathrm{N}, 48^{\circ} 52.79^{\prime} \mathrm{E}, 1212 \mathrm{~m}$, on Quercus sp., $20^{\top}, 1$ 우 (Yellow pan trap).

DISTRIBUTION. Type locality: France: "Corsica”. Palaearctic China, France, Italy, Tajikistan, Turkey. New record for Iran.

Tachytrechus notatus (Stannius, 1831)

MATERIAL. Markazi province: Arak, 19.VI.16, 3404.67' N, $49^{\circ} 43.36^{\prime} \mathrm{E}, 1643 \mathrm{~m}, 11{ }^{\circ}$ (Yellow pan trap); Arak, 22.VI.16, 3404.67'

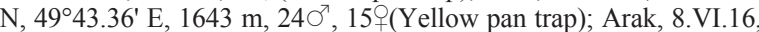
$34^{\circ} 04.67^{\prime} \mathrm{N}, 49^{\circ} 43.36^{\prime} \mathrm{E}, 1643 \mathrm{~m}$, on Juncus sp. and Avena sativa

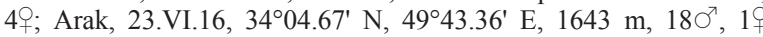
(Yellow pan trap); Lorestan province: Khak Patieh, 11.VII.16,

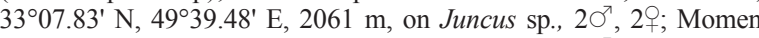

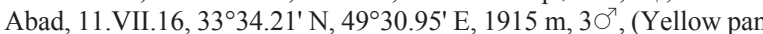
trap); Momen Abad, 11.VII.16, 3334.21' N, 49³0.95' E, 1915 m, on

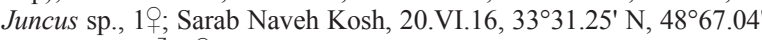

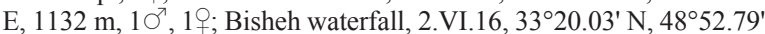
E, $1212 \mathrm{~m}$, on Quercus sp., 30', (Yellow pan trap).

DISTRIBUTION. Type locality: Germany: "Hamburg". Caucasus: Abkhazia, Armenia, Russia (Adygea, Chechnya, Kabardino-Balkaria, Krasnodar Territory). Europe, North Africa, Asia (Iran, Israel, Syria, Turkey, Turkmenistan, Siberia).

Subfamily Hydrophorinae Lioy, 1864 Hydrophorus Fallén, 1823

Hydrophorus balticus (Meigen, 1824)

MATERIAL. Markazi province: Hossein Abad, 19.V.16, 34 01.43' N, 49 46.28' E, 1915 m, on Juncus sp. and Equistum sp., 1; Baghdadi, 19.V.16, $34^{\circ} 01.75^{\prime}$ N, 49 45.21' E, 2017 m, on Populus sp. (Trees), $2 \sigma^{\top}, 2+$.

DISTRIBUTION. Type locality: Germany: Hamburg. Caucasus: Armenia, Azerbaijan, Georgia, Russia (Adygea, Alania, Chechnya, Kabardino-Balkaria, Karachai-Cherkessia, Krasnodar Territory). Europe, North Africa, Asia (Afghanistan, Algeria, Cyprus, Iran, Israel, Mongolia, Turkey, Siberia).

Subfamily Medeterinae Lioy, 1864

Medetera Fischer von Waldheim, 1819

Medetera jacula (Fallén, 1823)

MATERIAL. Lorestan province: Khak Patieh, 3.VI.16, $33^{\circ}$ 07.83' N, 49³9.48' E, $2024 \mathrm{~m}, 20^{7}$.

DISTRIBUTION. Type locality: Sweden: Scania. Caucasus: Armenia, Azerbaijan, Georgia, Russia (Alania, Chechnya, Kabardino-Balkaria, Krasnodar Territory, Rostov Region, Stavropol Territory). Europe, North Africa, Asia (Iran, Kazakhstan, Turkey, Siberia).

\section{Medetera meridionalis Negrobov, 1967}

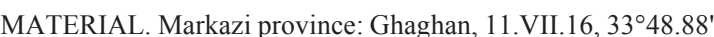
$\mathrm{N}, 49^{\circ} 29.88^{\prime} \mathrm{E}, 2070 \mathrm{~m}$, on Juncus sp., $2 \mathrm{\sigma}^{\top}, 1$, ; Lorestan province:

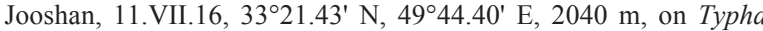
sp., $1 \sigma^{\top}, 1$.
DISTRIBUTION. Type locality: Russia: Voronezh, near Novokhopersk river. Caucasus: Armenia, Azerbaijan, Georgia, Russia (Krasnodar Territory, Rostov Region). Europe, Asia (Iran, Kazakhstan, Siberia, Turkey).

\section{Medetera pallipes (Zetterstedt, 1843)}

Fig. 8.

MATERIAL. Markazi province: Arak, 22.VI.16, 340․ $047^{\prime} \mathrm{N}$, 49 $43.36^{\prime} \mathrm{E}, 1643 \mathrm{~m}, 70^{\prime}, 14 ㅇ$ (Yellow pan trap).

DISTRIBUTION. Type locality: Scania, “in Ostrog ad Wadstena; Botnia orientali ad Johannis Ro prope Torneä" [Sweden; Denmark]. Caucasus: Georgia, Russia (Chechnya, Kabardino-Balkaria, Krasnodar Territory, Stavropol Territory). Europe, North Africa, Asia (Iran, Israel, Turkey). New record for Iran.

Thrypticus Gerstaecker, 1864

Thrypticus bellus Loew, 1869

MATERIAL. Lorestan province: Aligoudarz, 11.VII.16, $33^{\circ}$ $22.59^{\prime} \mathrm{N}, 4^{\circ} 43.39^{\prime} \mathrm{E}, 2024 \mathrm{~m}$, on Typha sp., 620', 38웅 Jooshan, 11.VII.16, $33^{\circ} 21.43^{\prime} \mathrm{N}, 49^{\circ} 44.40^{\prime} \mathrm{E}, 2040 \mathrm{~m}$, on Typha sp., $4 \mathrm{O}^{\top}, 1$ '

DISTRIBUTION. Type locality: UK: "Kew". Caucasus: Abkhazia, Armenia, Azerbaijan, Russia (Krasnoyarsk, Leningrad, Rostov Region, Vladivostok, Voronezh). Europe, North and Tropical Africa, Asia (Palaearctic China, Iran, Israel, Kazakhstan, Russian Far East, Siberia, Turkey.

\section{Subfamily Rhaphiinae Bigot, 1852 Rhaphium Meigen, 1803 \\ Rhaphium albifrons Zetterstedt, 1843}

MATERIAL. Lorestan province: Choghadoon, 1.VI.16, $33^{\circ}$ 28.77' N, 49 $10.37^{\prime} \mathrm{E}, 1580 \mathrm{~m}$, on Poaceae, $10^{7}$.

DISTRIBUTION. Type locality: Norway: "Scandinavia boreali-Norvegia Garnaes Vaerdaliae". Caucasus: Azerbaijan, Georgia, Russia (Krasnodar Territory). Europe, Asia (Afghanistan, Iran, Russian Far East, Siberia, Turkey, Uzbekistan).

\section{Rhaphium antennatum (Carlier, 1835)}

MATERIAL. Lorestan province: Jooshan, 11.VII.16, 33²1.43' N, 4944.40' E, $2040 \mathrm{~m}$, on Typha sp., $10^{2}$.

DISTRIBUTION. Type locality: Belgium: "Kimkempois pres de liege". Caucasus: Russia (Krasnodar Territory). Europe, Asia (Iran).

Rhaphium appendiculatum Zetterstedt, 1849

MATERIAL. Lorestan province: Khak Patieh, 3.VI.16, 33ํำ.83' N, 4939.48' E, $2024 \mathrm{~m}, 1 \mathrm{O}^{\top}$.

DISTRIBUTION. Type locality: Sweden: Scania ad Esperod. Caucasus: Abkhazia, Georgia, Russia (Adygea, Alania, Krasnodar Territory). Europe, North Africa, Asia (Afghanistan, Iran, Turkey, "Middle Asia").

Subfamily Sympycninae Aldrich, 1905

Campsicnemus Haliday, 1851

Campsicnemus armatus (Zetterstedt, 1849) Fig. 2.

MATERIAL. Lorestan province: Momen Abad, 11.VII.16, 3334.21' N, 49³0.95' E, $1915 \mathrm{~m}, 10^{\circ}$, (Yellow pan trap).

DISTRIBUTION. Type locality: Denmark: Rosenthal, Gryphium. Europe, North and Tropical Africa, Asia (Mongolia, Russian Far East, Siberia, Turkey). New record for Iran.

REMARK. The male trapped in Iran is remarkably similar to a subspecies C. armatus caffer Curran, 1926 (examined), known from southern Africa, and to $C$. a. deserti Vaillant, 1953 (unavailable name), described from Algeria. 


\section{Campsicnemus curvipes (Fallen, 1823)}

MATERIAL. Markazi province: Panj Ali, 22.V.16, 3402.27'

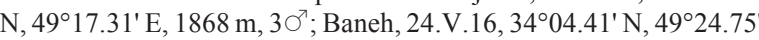
E, 2021 m, on Cannabis sp., 1; Lorestan province: Khaian, 13.V.16, $33^{\circ} 47.49^{\prime} \mathrm{N}, 48^{\circ} 54.44^{\prime} \mathrm{E}, 1676 \mathrm{~m}$, on Hordeum murinum near

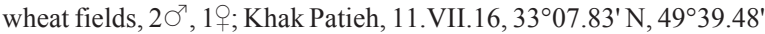
E, $2061 \mathrm{~m}$, on Poaceae and Typha sp., 1 . .

DISTRIBUTION. Type locality: not given [Europe]. Caucasus: Abkhazia, Armenia, Azerbaijan, Russia (Adygea, Alania, Dagestan, Kabardino-Balkaria, Karachai-Cherkessia, Krasnodar Territory, Stavropol Territory). Europe, North Africa, Asia (Iran, Siberia, Turkey).

\section{Campsicnemus magius (Loew, 1845)}

Fig. 1.

MATERIAL. Lorestan province: Sarab Dooreh, 20.VI.16, $33^{\circ}$ 46.91' N, 48 46.87' E, 1123 m, $10^{\prime}$.

DISTRIBUTION. Type locality: Italy: Sicily. Caucasus: Azerbaijan, Russia (Kabardino-Balkaria, Krasnodar Territory, Rostov Region). Europe, North Africa, Asia (Israel, Tajikistan, Turkey, Turkmenistan, Uzbekistan). New record for Iran.

\section{Campsicnemus tomkovichi Grichanov, 2009}

Fig. 3.

MATERIAL. Markazi province: Baghdadi, 19.V.16, 3401.75' N, 49 $45.21^{\prime}$ E, $2017 \mathrm{~m}$, on Populus sp. (Trees), $10^{\prime}$.

DISTRIBUTION. Type locality: Azerbaijan: Yardimli [district], Kreki. Caucasus: Armenia, Azerbaijan. New record for Iran.

\section{Campsicnemus umbripennis Loew}

MATERIAL. Markazi province: Mohajeran, 24.V.16, 3404.74' $\mathrm{N}, 49^{\circ} 25.69^{\prime} \mathrm{E}, 2017 \mathrm{~m}, 2$; ; Adeshteh, 27.V.16, 3405.10' N, $49^{\circ} 26.82^{\prime}$ E, $2159 \mathrm{~m}, 10^{7}$, 1웅 Baghdadi, 8.VI.16, 3402.05' N, $49^{\circ} 45.11^{\prime}$ E, $2000 \mathrm{~m}$, on Juncus sp. and Equistum sp., 1 1 ; Baghdadi, 19.V.16, $34^{\circ} 01.75^{\prime} \mathrm{N}, 49^{\circ} 45.21^{\prime} \mathrm{E}, 2017 \mathrm{~m}$, on Populus sp. (Trees);

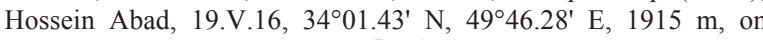
Juncus sp. and Equistum sp., 10', 9 ㅇ.

DISTRIBUTION. Type locality: Austria. Caucasus: Abkhazia, Armenia, Azerbaijan, Georgia, Russia (Adygea, Alania, Kabardino-Balkaria, Karachai-Cherkessia, Krasnodar Territory). Europe, North Africa, Asia (Afghanistan, Iran, Iraq, Israel, Tajikistan, Turkey, Turkmenistan).

\section{Lamprochromus Mik, 1878 \\ Lamprochromus sp.}

MATERIAL. Markazi province: Seyed Esmaeel road, 22.V.16, $34^{\circ} 13.68^{\prime} \mathrm{N}, 49^{\circ} 08.77^{\prime} \mathrm{E}, 2073 \mathrm{~m}, 10^{\top}, 19$. New record of the genus for Iran.

\section{Micromorphus Mik, 1878 \\ Micromorphus sp.}

MATERIAL. Markazi province: Arak, 23.VI.16, 340․ $047^{\prime} \mathrm{N}$ $49^{\circ} 43.36^{\prime} \mathrm{E}, 1643 \mathrm{~m}, 1$ ( (Yellow pan trap). New record of the genus for Iran.

\section{Sympycnus Loew, 1857}

Sympycnus pulicarius (Fallén, 1823)

MATERIAL. Lorestan province: Darband, 1.VI.16, 33²6.45'

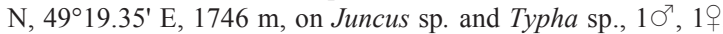

DISTRIBUTION. Type locality: not given [Sweden]. Caucasus: Azerbaijan, Russia (Alania, Kabardino-Balkaria, Karachai-Cherkessia, Stavropol Territory). Europe, Asia (Iran, Kazakhstan, Turkey, Siberia); Nearctic Region.

\section{Sympycnus simplicipes Becker, 1908}

MATERIAL. Lorestan province: Bisheh waterfall, 2.VI.16, $33^{\circ} 20.03^{\prime} \mathrm{N}, 48^{\circ} 52.79^{\prime} \mathrm{E}, 1212 \mathrm{~m}$, on Poaceae and Quercus sp., 2 \%

DISTRIBUTION. Type locality: Spain: Canary Is., Tenerrife. Caucasus: Abkhazia, Azerbaijan, Russia (Adygea, Krasnodar Territory). Asia (Iran, Iraq, Israel, Kazakhstan, Korea, Tajikistan, Turkey, Uzbekistan), Afrotropical and Oriental Regions.

\section{Syntormon Loew, 1857}

Syntormon aulicus (Meigen, 1824)

MATERIAL. Lorestan province: Khak Patieh, 11.VII.16, $33^{\circ} 07.83^{\prime} \mathrm{N}, 49^{\circ} 39.48^{\prime} \mathrm{E}, 2061 \mathrm{~m}$, on Poaceae and Typha sp., $10^{7}$.

DISTRIBUTION. Type locality: not given. Caucasus: Azerbaijan. Europe, North Africa, Asia (Iran, Turkey, "Middle Asia").

\section{Syntormon denticulatus (Zetterstedt, 1843)}

MATERIAL. Markazi province: Nahremian, 13.V.16, 33⒌ $54.26^{\prime}$ $\mathrm{N}, 4^{\circ} 00.57^{\prime}$ E, $2029 \mathrm{~m}$, on Juncus sp., 10'; Hossein Abad, 19.V.16, $34^{\circ} 01.43^{\prime} \mathrm{N}, 49^{\circ} 46.28^{\prime} \mathrm{E}, 1915 \mathrm{~m}$, on Juncus sp. and Equistum sp., 1; Baghdadi, 19.V.16, $34^{\circ} 01.75^{\prime} \mathrm{N}, 49^{\circ} 45.21^{\prime} \mathrm{E}, 2017 \mathrm{~m}$, on

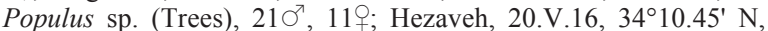
$49^{\circ} 32.10^{\prime}$ E, $1926 \mathrm{~m}$, on Poaceae and Equistum sp., $10^{7}$; Eijan, 22.V.16, $34^{\circ} 16.89^{\prime} \mathrm{N}, 49^{\circ} 17.46^{\prime} \mathrm{E}, 1765 \mathrm{~m}, 20^{\top}, 3$ ' ; Arak, 08.VI.16, $34^{\circ} 04.67^{\prime} \mathrm{N}, 49^{\circ} 43.36^{\prime} \mathrm{E}, 1643 \mathrm{~m}$, on Juncus sp. and Avena sativa,

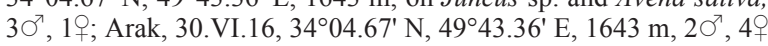
(Yellow pan trap); Ghaghan, 11.VII.16, 33 $48.88^{\prime} \mathrm{N}, 49^{\circ} 29.88^{\prime} \mathrm{E}$, $2070 \mathrm{~m}$, on Juncus sp., 2 ?

DISTRIBUTION. Type locality: Sweden: "Scania". Caucasus: Abkhazia, Armenia, Azerbaijan, Russia (Adygea, N Ossetia-Alania, Kabardino-Balkaria, Stavropol Territory). Europe, "North Africa", Asia (Afghanistan, Iran, Israel, Kyrgyzstan, Tajikistan, Turkey).

\section{Syntormon fuscipes (von Roser, 1840)}

Fig. 7.

MATERIAL. Markazi province: Seyed Esmaeel road, 22.V.16, $34^{\circ} 13.68^{\prime} \mathrm{N}, 49^{\circ} 08.77^{\prime} \mathrm{E}, 2073 \mathrm{~m}, 1^{\circ}$; Lorestan province: Khak

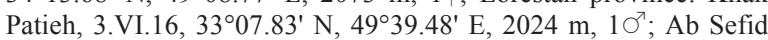
waterfall, 03.VI.16, $33^{\circ} 02.33^{\prime} \mathrm{N}, 4^{\circ} 36.05^{\prime}$ E, $2044 \mathrm{~m}$, on Juncus

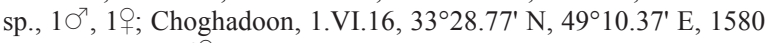
$\mathrm{m}$, on Poaceae, 4 ?

DISTRIBUTION. Type locality: "not given" (Germany: Württemberg). Caucasus: Abkhazia, Russia (Krasnodar Territory). Europe, Asia (Turkey), Afrotropics. New record for Iran.

\section{Syntormon pallipes (Fabricius, 1794)}

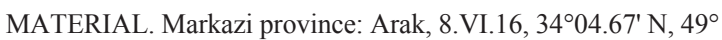
43.36' E, $1643 \mathrm{~m}$, on Juncus sp. and Avena sativa, 10 $10^{7}$; Baghdadi, 8.VI.16, $34^{\circ} 02.05^{\prime} \mathrm{N}, 49^{\circ} 45.11^{\prime} \mathrm{E}, 2000 \mathrm{~m}$, on Juncus sp. and

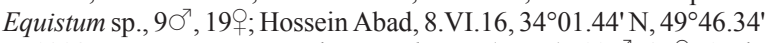
E, $1908 \mathrm{~m}$, on Juncus sp. under Populus sp. (Trees), 100', 279; Arak, 30.VI.16, 34 $4^{\circ} 04.67^{\prime} \mathrm{N}, 49^{\circ} 43.36^{\prime} \mathrm{E}, 1643 \mathrm{~m}, 10^{\top}$, (Yellow pan trap); Ghaghan, 11.VII.16, 33 $48.88^{\prime} \mathrm{N}, 49^{\circ} 29.88^{\prime} \mathrm{E}, 2070 \mathrm{~m}$, on Juncus

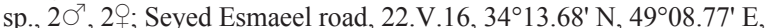

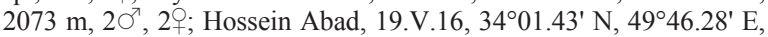

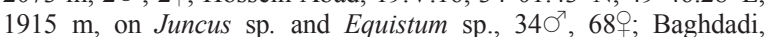
19.V.16, $34^{\circ} 01.75^{\prime} \mathrm{N}, 49^{\circ} 45.21^{\prime} \mathrm{E}, 2017 \mathrm{~m}$, on Populus sp. (Trees),

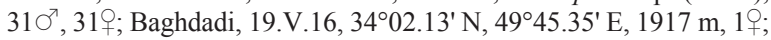
Ebrahim Abad, 19.V.16, $34^{\circ} 07.02^{\prime} \mathrm{N}, 50^{\circ} 02.38^{\prime} \mathrm{E}, 1712 \mathrm{~m}$, on

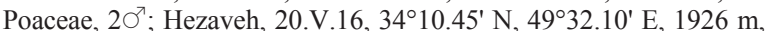

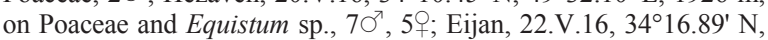

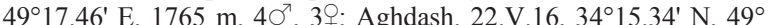

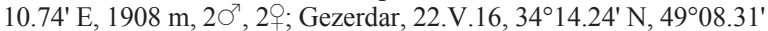

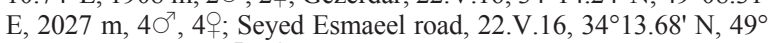
08.77' E, 2073 m, $20^{\prime}$, 2. ; Gezerdar, 22.V.16, 34 ${ }^{\circ} 12.50^{\prime} \mathrm{N}, 49^{\circ} 08.14^{\prime}$ E, 2255 m, 1우 Hosseinieh, 22.V.16, $34^{\circ} 15.58^{\prime} \mathrm{N}, 4^{\circ} 08.23^{\prime}$ E, 2010 
m, $20^{\top}, 2^{\circ}$; Hosseinieh, 22.V.16, 34 ${ }^{\circ} 17.28^{\prime} \mathrm{N}, 4^{\circ} 05.91^{\prime} \mathrm{E}, 2023 \mathrm{~m}$,

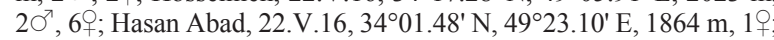

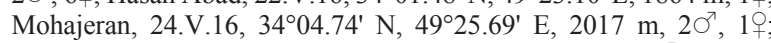

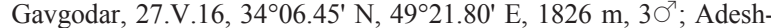

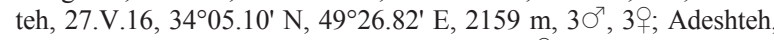
27.V.16, 34 $05.10^{\prime} \mathrm{N}, 49^{\circ} 26.82^{\prime} \mathrm{E}, 2159 \mathrm{~m}, 2^{\circ}$; Lorestan province: Khaian, 13.V.16, 33⒋49' N, 48 $54.44^{\prime}$ E, 1676 m, on Hordeum

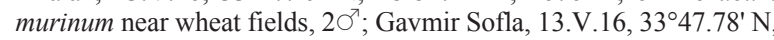

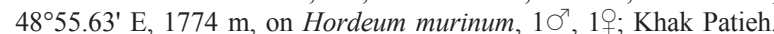

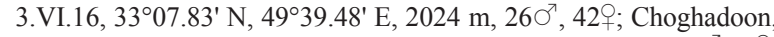
1.VI.16, $33^{\circ} 28.77^{\prime} \mathrm{N}, 49^{\circ} 10.37^{\prime} \mathrm{E}, 1580 \mathrm{~m}$, on Poaceae, $10^{\top}, 1$; Bisheh waterfall, 2.VI.16, $33^{\circ} 20.03^{\prime} \mathrm{N}, 4^{\circ} 52.79^{\prime} \mathrm{E}, 1212 \mathrm{~m}$, on Poaceae and Quercus sp., $10^{7}, 1^{\circ}$; Khak Patieh, 3.VI.16, 33 ${ }^{\circ} 07.83^{\prime} \mathrm{N}$, 4939.48' E, 2024 m, 30'; Momen Abad, 11.VII.16, 33³4.21' N, $49^{\circ}$ $30.95^{\prime}$ E, $1915 \mathrm{~m}$, on Juncus sp., $10^{7}$; Jooshan, 11.VII.16, 33²1.43'

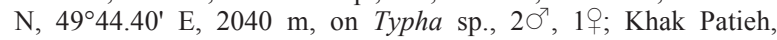
11.VII.16, 33 $07.83^{\prime} \mathrm{N}, 49^{\circ} 39.48^{\prime} \mathrm{E}, 2061 \mathrm{~m}$, on Poaceae and Typha

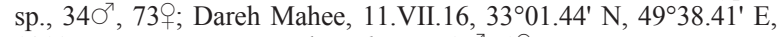
$1810 \mathrm{~m}$, on Juncus sp. and Typha sp., 10, 1 .

DISTRIBUTION. Type locality: Germany. Caucasus: Abkhazia, Armenia, Azerbaijan, Georgia, Russia (Adygea, Alania, Kabardino-Balkaria, Karachai-Cherkessia, Krasnodar Territory, Rostov Region). Europe, North Africa, Asia (Afghanistan, Palaearctic China, Iran, Iraq, Israel, Jordan, Kyrgyzstan, Tajikistan, Turkey, Uzbekistan); Oriental China; Afrotropics.

\section{Teuchophorus Loew, 1857 Teuchophorus sp.}

MATERIAL. Gavgodar, 10-Sep-15, 3406.45' N, $49^{\circ} 21.80$ E, 1826 m, on Typha sp., Salvia sp., Medicago sativa and Salix sp., 1 ㅇ.

\section{Discussion}

As a result of 2016 survey conducted in the two provinces, a new material of Dolichopodidae was collected and identified, belonging to 18 genera and 38 (including 5 unnamed) species listed above. The present research gives new records from the Central Iran, including Chrysotus pennatus, Sybistroma impar, Tachytrechus beckeri, Medetera pallipes, Campsicnemus armatus, C. magius, C. tomkovichi and Syntormon fuscipes found for the first time in Iran.

The identified species of the family Dolichopodidae were sampled at the height of 1123-2255 m above sea level. Most collected species are widespread across the Palaearctic Region. According to sampling data, three species $(S$. pallipes, $P$. regalis and $T$. notatus) have the highest total abundance. Two species (not listed) represent apparently new for science species to be described later. As a result of our investigation, the number of reported species from Iran has reached now to 131 species. We suggest that many more species will be revealed in Iran, if new districts and localities are investigated with the use of mass trapping methods.

ACKNOWLEDGEMENTS. We are grateful to Dr. Akram Ahmadi (Baran Plant Protection Institute, Arak, Iran) for the identification of plant species. The work of IYG is partly supported by the Russian Foundation for Basic Research grant N 14-04-00264-a.

\section{References*}

Ahmadi A., Gheibi M., Ostovan H., Hesami S., Grichanov I.Ya. 2016 (accepted). New records of Dolichopodidae (Diptera) from Central Provinces of Iran // Halteres. Vol.7. P.191-196.

Grichanov I.Ya. 2007. A checklist and keys to Dolichopodidae (Diptera) of the Caucasus and East Mediterranean. St.Petersburg: VIZR. P.1-160 (Plant Protection News Supplements).

Grichanov I.Ya. 2014. Alphabetic list of generic and specific names of predatory flies of the epifamily Dolichopodoidae (Diptera). St.Petersburg: VIZR. P.1-544 (Plant Protection News Supplements).

Grichanov I.Ya. 2016. Review of faunal investigation of predatory flies of the family Dolichopodidae (Diptera) in Iran // Acta Biologica Sibirica. Vol.2. No.4. P.11-14.

Grichanov I.Ya., Alikhani M., Rabieh M.M. 2010. New data on the distribution of Dolichopodidae (Diptera) in Iran // International Journal of Dipterological Research. Vol.21. No.3. P.195-201.

Khaghaninia S., Kazerani F., Talebi A.A., Pollet M. 2016. New records of Diaphorinae (Dip., Dolichopodidae) from North West Iran // 22nd Iranian Plant Protection Congress, Tehran University, Tehran, Iran. P.21-29.

Negrobov O.P., Selivanova O.V., Maslova O.O., Chursina M.A. 2013. Check-list of predatory flies of the family Dolichopodidae (Diptera) in the fauna of Russia // Grichanov I.Ya., Negrobov O.P. (eds.). Fauna and taxonomy of Dolichopodidae (Diptera). Collection of papers. (Plant Protection News, Supplements). St.Petersburg: VIZR. P.47-93.

\footnotetext{
* For the complete Iranian reference list see Grichanov [2016].
} 